\title{
Lenhos fósseis do Neógeno da Bacia do Acre, Formação Solimões: contexto paleoambiental
}

\author{
Luciano Gandin Machado ${ }^{1,2}$, Rita Scheel-Ybert ${ }^{2}$, Robson Tadeu Bolzon ${ }^{3}$, \\ Marcelo de Araujo Carvalho ${ }^{2} \&$ Ismar de Souza Carvalho ${ }^{1}$
}

\begin{abstract}
Resumo Este trabalho aborda o estudo de lenhos fósseis coletados nas margens dos rios Acre, Furnaia, Juruá, Purus e Moa, em afloramentos da Formação Solimões (Mioceno superior), Bacia do Acre. A descrição anatômica e morfológica das estruturas celulares dos lenhos fósseis foi acompanhada de inferências sobre a preservação dos elementos constituintes dos lenhos, visando reunir dados tafonômicos e parâmetros paleoambientais regionais. Os resultados indicam que a sílica é o principal mineral constituinte dos lenhos, ocorrendo também inclusões de óxidos de ferro. O conjunto de características anatômicas incluindo vasos de diâmetro médio (100-200 $\mu \mathrm{m})$ a grande $(\geq 200 \mu \mathrm{m})$, predominância de vasos solitários, poucos vasos por milímetro quadrado e placas de perfuração simples, possibilitou inferir um clima tropical para o Acre no final do Mioceno. A presença de anéis de crescimento e vasos com tendência a semiporosidade nos exemplares, sugere uma variação na disponibilidade hídrica indicando um clima sazonal, com uma estação seca. O registro da paleovegetação, através da palinologia, indica a presença de lagos de água doce e pântanos tropicais. Corpos de água permanentes (pântanos, lagos rasos) foram difundidos durante a deposição da Formação Solimões, indicando que a Amazônia sul-ocidental agiu como uma bacia plana similar ao que é nos dias de hoje o Sistema do Pantanal, mas alimentada pelos Andes. Os lenhos fósseis possuem afinidade botânica com as famílias Caesalpinioideae (Fabaceae), Lythraceae e Myrtaceae, e se relacionariam à regiões associadas a este paleoambiente.
\end{abstract}

Palavras-chave: Lenhos fósseis, Amazônia brasileira, Mioceno, Bacia do Acre, Formação Solimões.

\begin{abstract}
Fossil woods from Neogene of Acre Basin, Solimões Formation: paleoenvironmental context. This paper discusses the study of fossil wood collected along the banks of Acre, Furnaia, Juruá, Purus and Moa rivers, located in the outcrops of Solimões Formation (Upper Miocene), Acre Basin. The anatomical and morphological description of the cellular structures was accompanied by inferences about the preservation of the woods constituent elements, aiming to bring together taphonomic data and palaeoenvironmental regional parameters. The results indicate silica as the major component of the fossils, also occurring inclusions of iron oxides. The group of anatomical features includes vessels of medium (100 to $200 \mu \mathrm{m})$ to large ( $\geq 200 \mu \mathrm{m}$ ) diameter, with a predominance of solitary vessels, few vessels per square millimeter and simple perforation plates. The presence of these characters enabled to infer a tropical climate in Acre at the late Miocene. The presence of growth rings and vessels trending to semi-porosity suggests a variation in water availability, which indicates a seasonal climate with a dry season. The palynological record found in the literature indicates a palaeovegetation with the presence of freshwater lakes and tropical marshes. Permanent water bodies (wetlands, shallow lakes) were frequent during the deposition of the Solimões Formation, indicating that the Amazon south-western basin acted as a plane basin, similar to the system of the modern Pantanal, but fed by the Andes. The fossil woods have affinity with the recent botanical families Caesalpinioideae (Fabaceae), Lythraceae and Myrtace, which can be related to this palaeoenvironment.
\end{abstract}

Keywords: Fossil wood, Brazilian Amazon, Miocene, Acre Basin, Solimões Formation.

INTRODUÇÃO A região sul-ocidental da Amazônia é explorada desde o início do século 20 por expedições geológicas que coletaram e catalogaram fósseis, como as realizadas por: Pedro de Moura, em 1936, e Llewellyn Ivor Price e George Gaylord Simpson, em 1956. Muitas localidades fossilíferas foram registradas no Estado do Acre pelo Projeto Radambrasil, em 1977. A maior parte dos fósseis é encontrada na Formação Solimões, que inclui: moluscos (Vonhof et al. 1998), peixes (Richter 1984, 1989), quelônios (Bocquentin \& Guilherme 1997), crocodilianos (Souza Filho 1998), aves (Alvarenga \& Guilherme 2003), mamíferos (Cozzuol et

1 - Departamento de Geologia, Instituto de Geociências, Centro de Ciências Matemáticas e Naturais, Universidade Federal do Rio de Janeiro, Rio de Janeiro (RJ), Brasil. E-mail: lucianogmachado@ufrj.br, ismar@geologia.ufrj.br

2 - Laboratório de Paleoecologia Vegetal, Museu Nacional, Universidade Federal do Rio de Janeiro, Rio de Janeiro (RJ), Brasil. E-mail: scheelybert@mn.ufrj.br; mcarvalho@mn.ufrj.br

3 - Departamento de Geologia, Setor de Ciências da Terra, Universidade Federal do Paraná, Curitiba (PR), Brasil. E-mail: bolzonrt@ufpr.br 
al. 2006), primatas (Kay \& Cozzuol 2006), ostracodes (Ramos et al. 2001), palinomorfos (Hoorn 1993, Silva et al. 2004, Latrubesse et al. 2007), folhas (Maury 1937) e madeiras fósseis (Mussa 1959, Mussa et al. 2002).

Neste estudo foi realizada a análise de seis lenhos fósseis coletados nas margens dos rios Acre, Furnaia, Juruá, Purus e Moa, em afloramentos da Formação Solimões (Mioceno superior), Bacia do Acre (Fig. 1). São apresentadas as descrições anatômica e morfológica das estruturas celulares dos lenhos fósseis, inferências sobre a preservação dos elementos constituintes dos exemplares, visando reunir dados tafonômicos, e parâmetros paleoecológicos, paleoambientais e paleoclimáticos regionais. Este trabalho é uma contribuição à Paleontologia, Geologia e Paleoflorística da região norte do Brasil, e almeja apresentar os lenhos fósseis como indicadores de parâmetros paleoambientais regionais, pois os poucos trabalhos voltados a esta compreensão (Frailey et al. 1988, Latrubesse et al. 1997, Latrubesse et al. 2007) estão focados nas análises palinológicas e de paleovertebrados.

\section{ÁREA DE ESTUDO}

Considerações geológicas e ação de eventos tectônicos sobre a Bacia do Acre A região sul-ocidental da Amazônia sofreu grandes mudanças geológicas, climáticas e ambientais ao longo de sua história. Um importante evento geológico registrado no Cretáceo Superior foi a orogenia Andina, que transformou a Bacia do Acre em marginal aberta para o tipo intracontinental no final do Cretáceo Superior e Paleógeno (Miura 1972). Esta orogenia mudou a direção da drenagem da bacia, o clima, a geologia, a vegetação e a fauna.

A Bacia do Acre (Fig. 2) apresenta uma área total $230.000 \mathrm{~km}^{2}$. É limitada à nordeste, pelo Arco de Iquitos, que a separa da Bacia do Solimões e à sudeste pelo Lineamento Madeira. Ao sul, a bacia é limitada pelo Escudo Brasileiro e à oeste e à noroeste pelo Lineamento Juruá. Estende-se pelo território peruano com o nome da Bacia de Paztazza (Miura 1972, Cavalcante 2006).

As seções do Cretáceo Superior e Paleógeno da Bacia do Acre, constituídas de sedimentos vermelhos continentais, são correlacionáveis com bacias da Colômbia, Peru e Equador (Miura 1972, Petri \& Fúlfaro 1983). O topo da Formação Solimões pode ser estratigraficamente correlacionado com as camadas do Mesopotamiano da Argentina (níveis mais baixos da Formação Itazaingó) e do Uruguai (Formação Kiyu), e

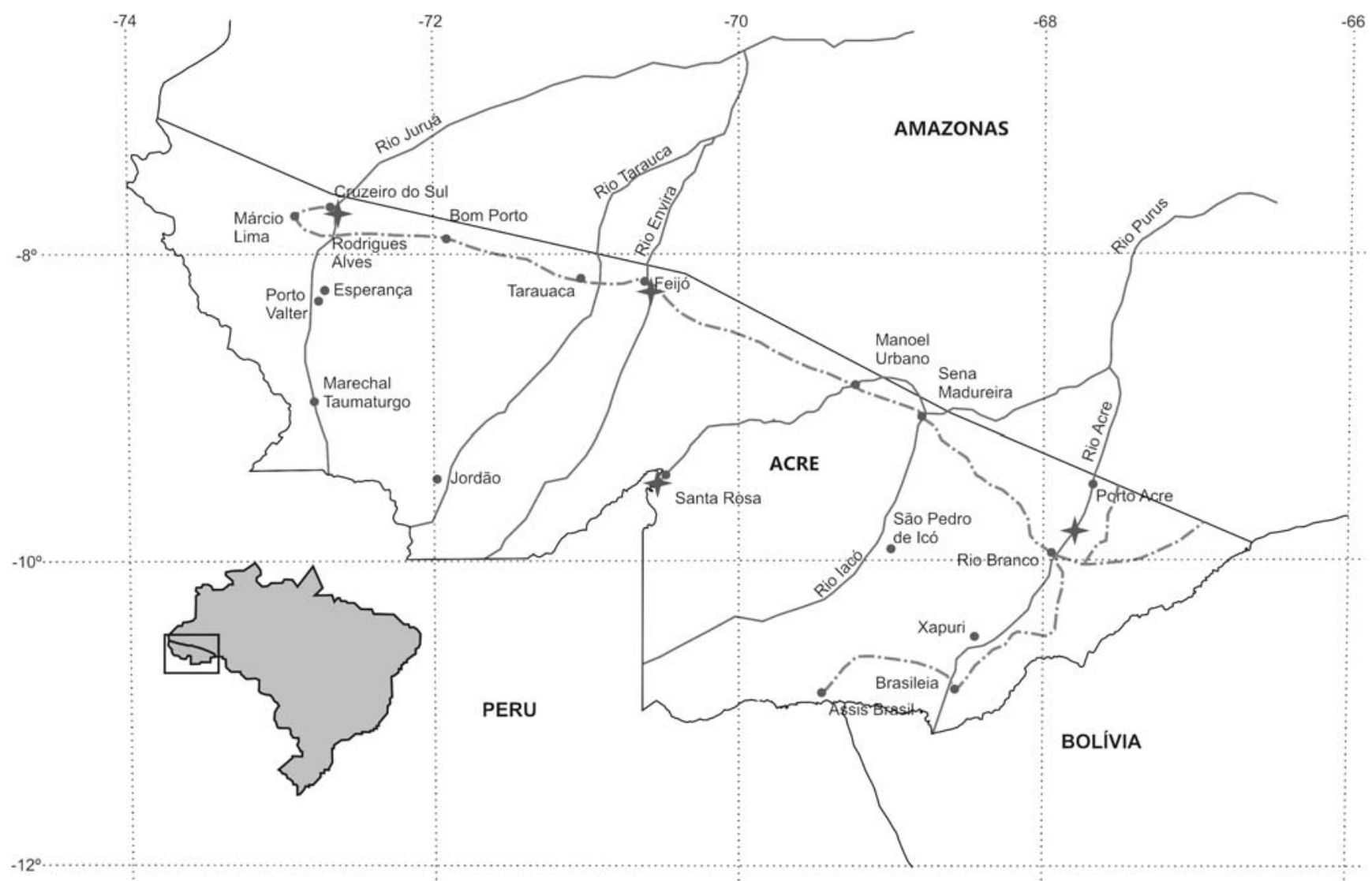

Figura 1 - Mapa rodoviário do Estado do Acre com os principais rios da bacia hidrográfica. As estrelas representam aproximadamente os pontos de coleta dos lenhos fósseis (modificado de DNIT 2002). 

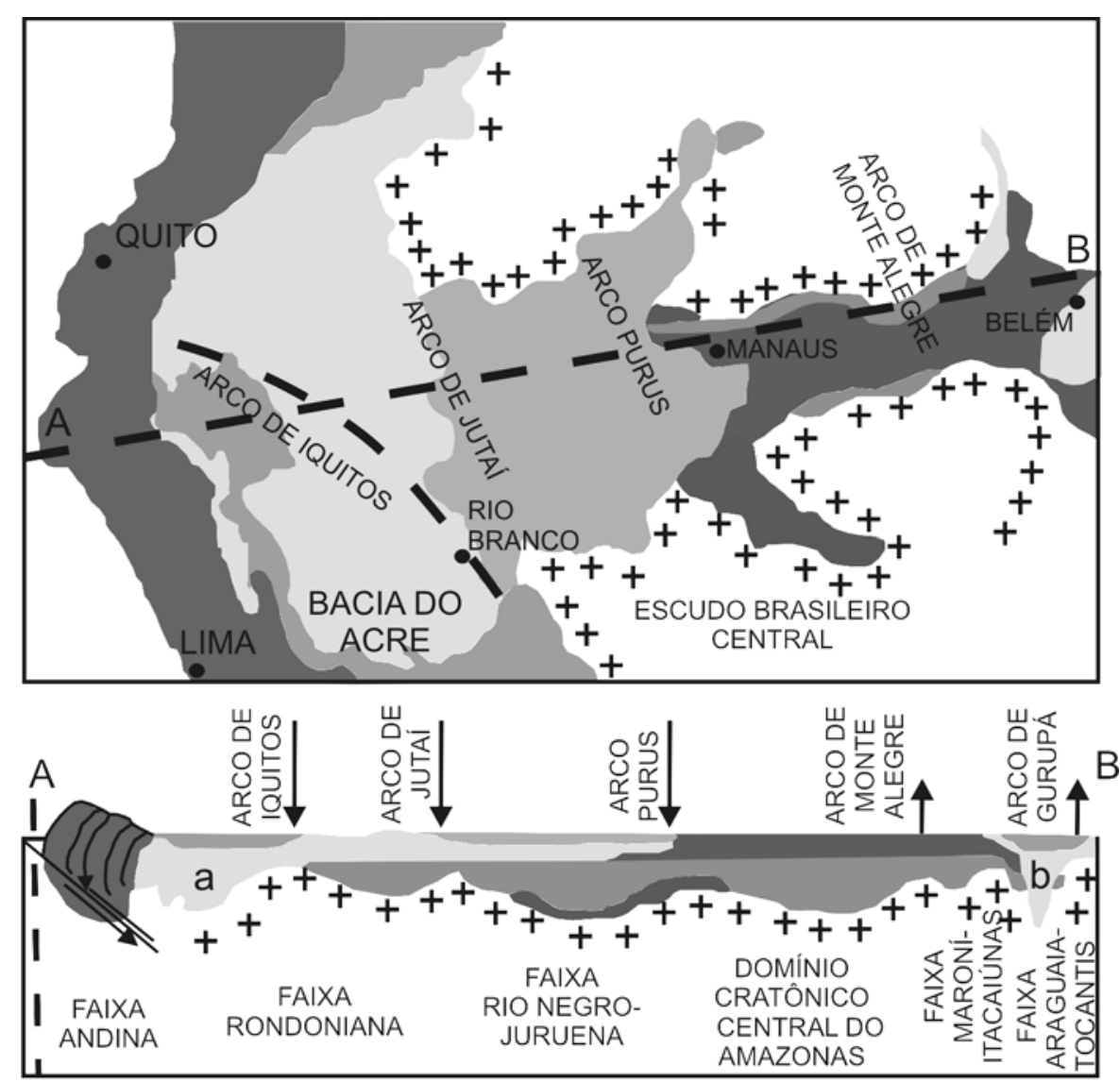

Figura 2 - Bacia do Acre e seu contexto geotectônico: 1) A - Formação Solimões, B-Sedimentos terciários. 2) Formação Içá. 3) Formação Alter do Chão. 4) Coberturas Proterozoicas. 5) Rochas Paleozoicas. 6) Quaternário. Modificado de Bezzera (2003).

também com a fossilífera Formação Urumaco da Venezuela (Latrubesse et al. 2007). Os terrenos cenozoicos do Acre e do Amazonas ocidental são pouco estudados, recebendo diferentes denominações para as unidades litoestratigráficas (Petri \& Fúlfaro 1983).

$\mathrm{Na}$ bacia estão presentes rochas sedimentares neopaleozoicas, mesozoicas e cenozoicas que são representadas pelas formações Apuí, Cruzeiro do Sul, Rio Moura, Juruá-Mirim, Moa, Rio Azul, Divisor, Ramon e Solimões (Feijó \& Souza 1994).

Os lenhos fósseis estudados foram todos coletados em margens de rios que cortam a Formação Solimões. A Formação Solimões é constituída litologicamente por argilitos cinzas e cinza-esverdeados, intercalados com bancos de arenito, camadas de linhito e gipsita (Oliveira \& Souza Filho 2001). Esta unidade apresenta estratificações plano-paralelas e cruzadas tabulares e acanaladas de pequena, média e grande amplitude (Cavalcante 2005).

Os primeiros dados palinológicos para a Bacia do Amazonas foram publicados por Daemon \& Contreiras
(1971), que sugeriram a idade Paleoceno-Mioceno para os sedimentos. Cruz (1984) estabeleceu três zonas palinológicas, Mioceno, Mioceno/Plioceno e Plioceno para perfurações do CPRM/DNPM. Hoorn (1993) analisou amostras palinológicas de duas perfurações no Estado do Amazonas próximas à divisa com o Peru, e identificou 5 biozonas polínicas para o Mioceno, do inicial ao médio/final. Silva et al. (2004) analisaram amostras palinológicas dos afloramentos Niterói e Patos e definiram a idade dos sedimentos como Mioceno, posteriormente, Latrubesse et al. (2007) estenderam estas análises para mais dois afloramentos da Formação Solimões ao longo do rio Acre (Niterói, Patos, Barranco da Elizete e Murici) determinando a idade como do Mioceno final.

Considerações paleoambientais sobre a Amazônia sul-ocidental no Mioceno Do início do Cretáceo até o Recente, o clima da Terra seguiu uma tendência ao resfriamento com pulsos de breves aquecimentos (Scotese 2003). A movimentação dos continentes do Gondwana eliminou a corrente Circum-equatorial que 
mantinha o continente Antártico quente. O surgimento do Istmo do Panamá e o estabelecimento da corrente Circumpolar Antártica, provavelmente durante o Plioceno, resultaram em importantes mudanças climáticas (Bolzon \& Marchiori 2002). O clima durante o Mioceno foi mais quente, com cinturões climáticos bem definidos do pólo para o Equador (Scotese 2003). As condições climáticas quente-temperadas no Mioceno foram intensificadas pelo soerguimento das modernas cadeias de montanha (Del Corro 1964; Scotese 2003). Os registros do Mioceno da América do Sul indicam uma flora similar à atual, tanto em regiões tropicais quanto nas temperadas frias (Bolzon \& Marchiori 2002).

Análises de isótopos estáveis em moluscos (Vonhof et al. 1998) sugerem para a Formação Solimões um paleoambiente com alguns lagos levemente salinos, em que a aridez foi fraca e insuficiente para produzir solos calcáreos ou evaporitos. Ao contrário, há relato que a ocorrência de veios de gipsita e material carbonático na Formação Solimões indicaria a presença de clima semiárido (Passos 2000). De acordo com a autora a origem do material carbonatado deve-se ao fato de que estes sais solúveis foram carregados pelos cursos d'água de fontes situadas à oeste da Bacia do Acre e acumulados em lagos, que devem ter sido submetidos a um clima árido capaz de provocar evaporação suficiente para formação destes evaporitos (Passos 2000). Räsänen et al. (1998) explicaram a abundância de níveis carbonosos, de fósseis e a predominância de sedimentos finos, ocasionalmente com precipitação química, através do contexto de um sistema fluvial que drenava a região de leste para oeste, em direção a uma área deprimida posicionada nas bordas da cadeia andina em processo de soerguimento (Orogenia Quéchua). Esta área também recebia sedimentação de alta energia sob a forma de leques aluviais provenientes do oeste da Cordilheira Andina.

Análises palinológicas, faunísticas e geológicas sugeriram que o clima da região sul-ocidental da Amazônia (Acre) durante o Mioceno final foi tropical úmido, com uma estação seca (Latrubesse et al. 2007). Uma fauna abundante, incluindo peixes, tartarugas, crocodilos e mamíferos, habitou uma associação de rios, pântanos, lagos e florestas de galerias (Latrubesse et al. 2007). O registro paleopalinológico indica a presença de lagos de água doce e pântanos tropicais (Latrubesse et al. 2007). Corpos d'água permanentes (pântanos, lagos rasos) foram distribuídos durante a deposição da Formação Solimões, indicando que a Amazônia sul-ocidental era uma bacia plana similar ao Sistema do Pantanal nos dias de hoje, mas alimentada pelos Andes (Latrubesse et al. 2007).

Oliveira \& Souza Filho (2001) sugeriram para a região, a partir de estudos de crocodiliformes, um ambiente deposicional fluvial meandrante e com lagos formados por canais abandonados. A assembleia fóssil de vertebrados analisada por Latrubesse et al. (2007) revelou importantes indicadores ambientais. Os roedores Kiyutherium aff. K. orientalis Francis \& Mones 1965 (Hydrochoeridae) habitavam áreas próximas a corpos d'água como as capivaras hoje. Outros mamíferos como Proterotheres (Liptoterna), Astrapotheres, Glyptodon, Pampatheres (Xenarthra, Cingulata), e preguiças de chão (Mylodontidae e Megatheriidae) indicam hábitos mais terrestres, sendo ambos pastadores. A presença de primatas Platyrrhini das famílias Cebidae e Atelidae, indica a existência de extensivas áreas de florestas de galeria ao longo dos rios. Os fósseis de vertebrados não sustentam a hipótese de um ambiente marinho, mesmo que os grupos mais ricos da fauna sejam aquáticos (crocodilianos, tartarugas e peixes). A tartaruga de rio da família Pelomedusidae, endêmica da América do Sul, é um bom indicador de corpos d'água estáveis com abundante vegetação e um clima tropical a subtropical. Ossos e cartilagens de peixes, répteis, cetáceos e peixe-boi indicam uma grande bacia de drenagem com lagos rasos e pântanos, cortados por cinturões fluviais. Alguns peixes como Arapiama, Hoplias, Colosoma e outros gêneros são encontrados hoje nos sistemas fluviais da bacia do Amazonas. Os siluriformes são os mais abundantes e diversificados grupos de peixes encontrados, e são bons indicadores de ambientes de água doce.

MATERIAL E MÉTODOS Os exemplares de lenhos fósseis estudados pertencem à Coleção do Laboratório de Pesquisas Paleontológicas da Universidade Federal do Acre (UFAC) (Pb168), à Coleção de Paleontologia do Departamento Nacional da Produção Mineral do Rio de Janeiro (NR404, NR570, NR573, NR491) e à Coleção de Paleontologia do Laboratório de Macrofósseis, Paleoinvertebrados e Paleobotânica do Departamento de Geologia da Universidade Federal do Rio de Janeiro (UFRJ-DG Pb746).

Na Coleção de Paleontologia do Departamento Nacional da Produção Mineral do Rio de Janeiro (DNPM-RJ), foram encontrados holótipos tombados no livro de registro de material paleobotânico e cerca de 45 lenhos tombados em três livros de registro de material paleobotânico e paleontológico geral.

Os procedimentos usados para a laminação basearam-se nos trabalhos com fósseis (Bolzon 1999; Hass \& Rowe 1999) e de petrografia (Norton \& Curchin 2002). A laminação consistiu das seguintes etapas: 1) seleção da região de corte, 2) redução, 3) impregnação, 4) secções, 5) acabamento, 6) coloração, 7) colagem e 8) desbaste final.

As descrições macro e microscópicas das lâminas delgadas dos três planos anatômicos da madeira 
seguiram, sempre que possível, as recomendações da Associação Internacional de Anatomistas da Madeira IAWA Committee (Wheeler et al. 1989).

\section{RESULTADOS E DISCUSSÃO Descrições anatômicas e identificação taxonômica dos lenhos fósseis}

\author{
Reino PLANTAE \\ Sub-reino VASCULARIOPHYTA \\ Divisão ANTHOPHYTA \\ Família FABACEAE (CAESALPINIOIDEAE) \\ Zollernioxylon $\mathrm{sp}$.
}

[Figuras 3 a 5]

MATERIAL EXAMINADO Brasil - Acre: Rio Juruá, Município Cruzeiro do Sul. Pedro de Moura (1935) (NR 404, DNPM-RJ). Brasil - Acre: Rio Furnaia, km 45 do paralelo $10^{\circ}$, Município de Feijó. Braulino de Carvalho (28.VI.1924) (NR 570, DNPM-RJ). Brasil Acre: Vale do Rio Moa, Município de Cruzeiro do Sul. (NR 573, DNPM-RJ).

DESCRIÇÃO MACROSCÓPICA Lenho de cor amarela a marrom-escuro (NR 404), de cor clara, com tons de cinza e branco (NR 570) ou tendendo ao marrom (NR 573). Em secção transversal, poros visíveis a olho $\mathrm{nu}$, sem arranjos preferenciais, solitários na maioria ou múltiplos de 2-3(4), alguns em cachos. Anéis de crescimento geralmente de difícil visualização a olho nu, mas podendo ser delimitados por parênquima marginal ou zona fibrosa sem vasos (NR 573). Raios muito finos, visíveis apenas em lupa. Parênquima em bandas regulares, às vezes confluentes a outras bandas, podendo ocorrer bandas irregulares com diâmetro variável. Em secção longitudinal tangencial, estratificação dos raios visível a olho nu. Individualização dos raios visível apenas em lupa, muito pequenos e baixos. Linhas vasculares longas, predominantemente lineares, pouco inclinadas em relação aos raios, de cores claras.

DESCRIÇÃO MICROSCÓPICA Anéis de crescimento com limites distintos, marcados pela presença de parênquima marginal e por uma redução do diâmetro dos poros e eventualmente da quantidade de poros. Lenho com porosidade difusa ou apresentando anel semiporoso. Poros predominantemente solitários $(67,3 \%)$, geminados e múltiplos de dois (30,7\%), raramente em cachos (2\%). Todos os vasos solitários de contorno arredondado. Diâmetro tangencial do lúmen dos vasos em média de $134,4 \mu \mathrm{m}$ (maior padrão de desvio $=47,4$; intervalo $=54,2-248,0 \mu \mathrm{m} ; \mathrm{n}=25)$ com muito poucos vasos por milímetro quadrado (média de 3-4,4; maior padrão de desvio $=1,7 ;$ intervalo $=1-8 ; \mathrm{n}=25)$. Elementos de vasos com comprimento de $354,7 \mu \mathrm{m}$ (maior padrão de desvio $=154,7 ;$ intervalo $=249,7-817,6 \mu \mathrm{m} ; \mathrm{n}=$ 5-20) e placas de perfuração simples. Pontoações intervasculares alternas, ornamentada. Parênquima em faixas longas com 3-5(7) células de largura, geralmente contínuas, regulares, às vezes irregulares, sinuosas e confluentes a outras faixas. Parênquima com 3-6 faixas por milímetro linear (média $=4,7$ ). Raios estratificados principalmente bisseriados $(79,4 \%$, sendo $17 \%$ em NR 573 ), com poucos raios unisseriados (7\%), raros trisseriados (2,3\%, sendo $70 \%$ em NR 573) e muito raros 4 -seriados $(0,6 \%$, em NR 404) e raros (9, em NR 573). Raios sub-homogêneos com células procumbentes e uma fileira marginal de células quadradas. Raios de comprimento baixo (média $=241,6 \mu \mathrm{m}$; maior padrão de desvio $=54,7 ;$ intervalo $89,8-348,4 \mu \mathrm{m} ; \mathrm{n}=25) \mathrm{com}$ 8,3 raios por milímetro linear (maior padrão de desvio $=2,5$; intervalo 5-12; $\mathrm{n}=10-25$ ). Séries cristalíferas presentes. Parênquima axial e fibras estratificadas.

COMPARAÇÃO As características anatômicas das amostras analisadas mais relevantes taxonomicamente são: estrutura estratificada, parênquima aliforme, pontoações guarnecidas, raios sub-homogêneos.

Apenas a família Fabaceae Lindl. reúne o conjunto das características distintivas. Esta família é dividida nas subfamílias Caesalpinioideae DC., Mimosoideae DC. e Faboideae Rudd. Os exemplares analisados distinguem-se das Mimosoideae, pois estas se caracterizam pela ausência de estratificação em todos os elementos e por apresentarem raios sempre homogêneos, compostos por células procumbentes (Barreta-Kuipers 1981). A subfamília Faboideae caracteriza-se pela estratificação regular de todos os elementos em cerca de $75 \%$ das espécies, enquanto que em Caesalpinioideae ocorrem em apenas cerca de 20\% (Wheeler \& Baas 1992). O exemplar fóssil apresenta características anatômicas que remetem ao gênero Zollernia, particularmente o parênquima aliforme disposto em longas faixas aproximadamente regulares. Os exemplares foram comparados com as espécies fósseis descritas por Mussa (1959) e como o exemplar descrito por Mussa et al. (2002).

As características anatômicas dos exemplares estudados permitiram atribuí-los ao gênero Zollernioxylon Mussa (1959). As diferenças apresentadas são decorrentes da plasticidade ambiental das estruturas anatômicas. Estas podem ser observadas nos anéis de crescimento praticamente ausentes na espécie tipo, conquanto bem demarcados por parênquima marginal e pela redução da frequência e diâmetro dos poros nos exemplares estudados. O diâmetro dos poros e o comprimento dos elementos de vasos também apresentaram leves variações. 

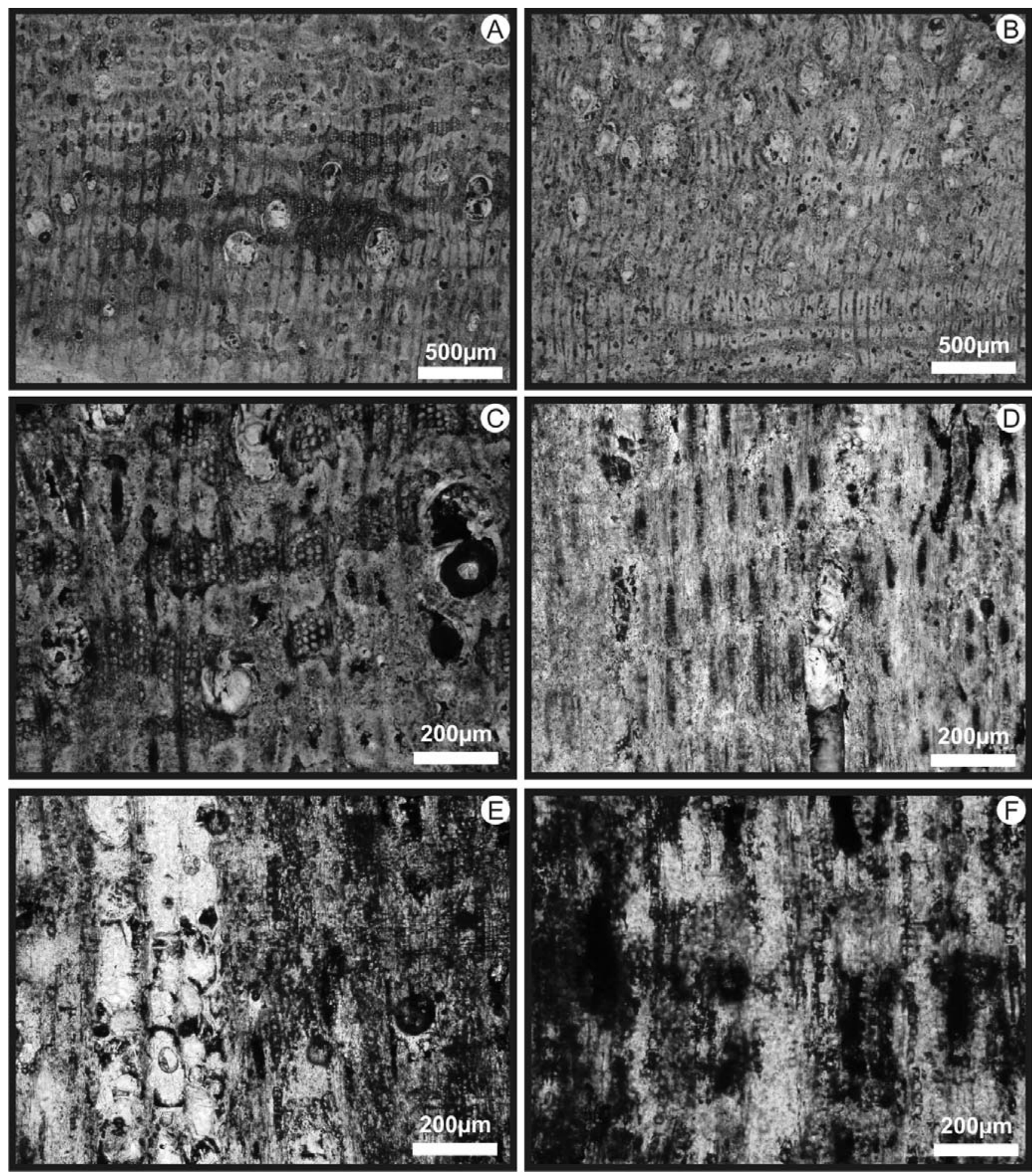

Figura 3 - Microfotografias em microscópio óptico de transmissão do exemplar NR404. A) Corte transversal, observa-se o parênquima axial paratraqueal em faixas largas, com mais de 3 células de espessura (3 a 5 células) e o parênquima marginal com poucas células de espessura. B) Corte transversal, com porosidade difusa tendendo a anel semiporoso. C) Corte transversal evidenciando as células do parênquima axial e fibras; variação na preservação celular. D) Corte longitudinal tangencial, com raios baixos; poucos unisseriados, na maioria bisseriados e escassos trisseriados e raros tetrasseriados; estratificados. E) Corte longitudinal radial, corpo do raio com células procubentes e fileira marginal de células quadradas. F) Corte longitudinal radial, séries cristaliferas. 

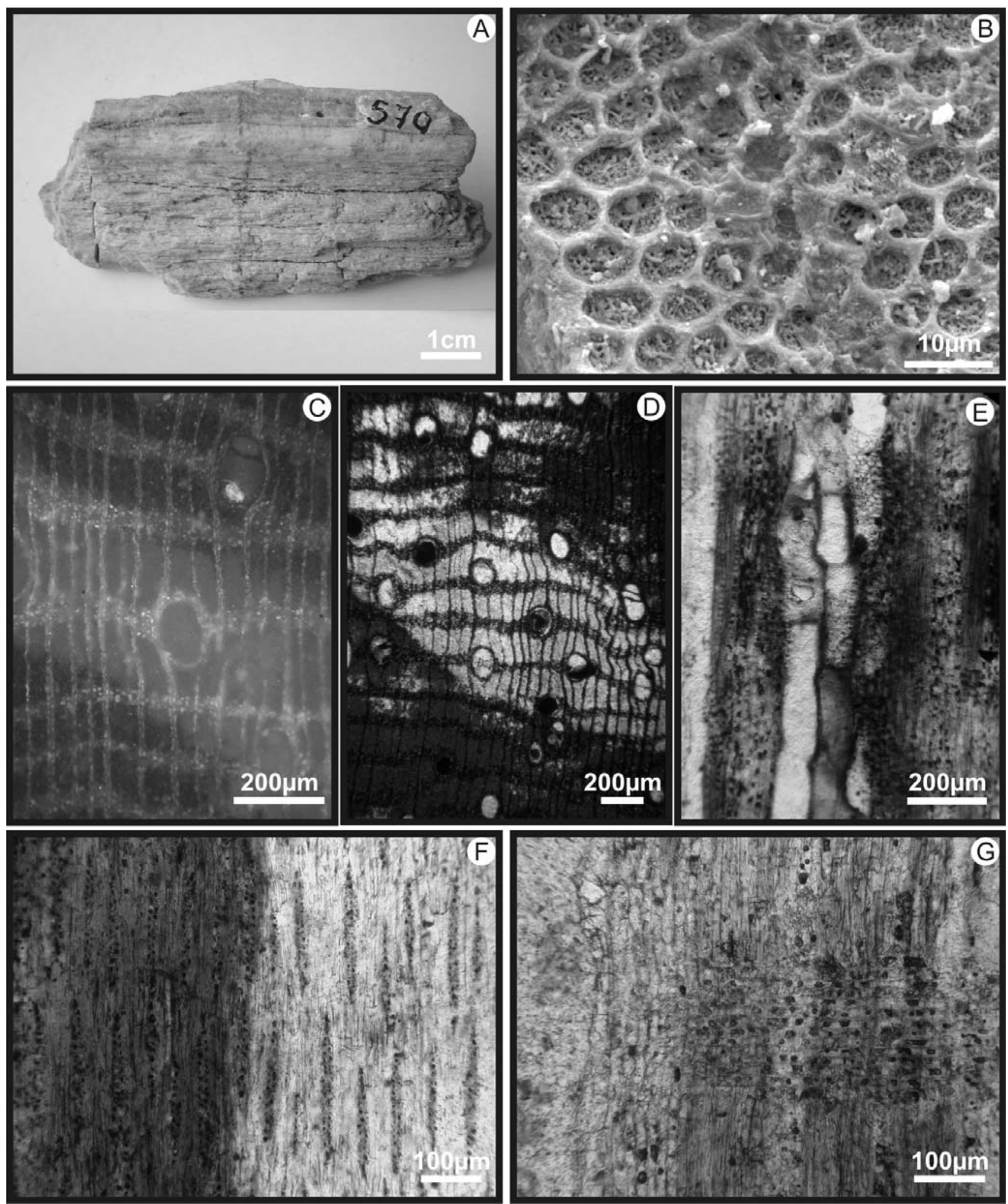

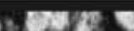

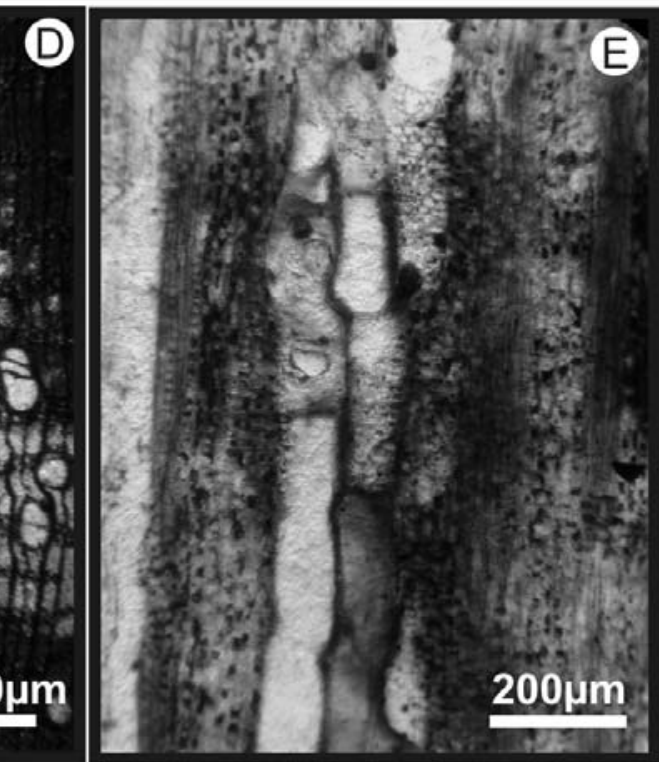
wives: Strpes: $x^{2}$

at: 1. 3 , th

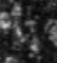

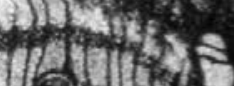

\section{(D)}

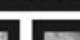

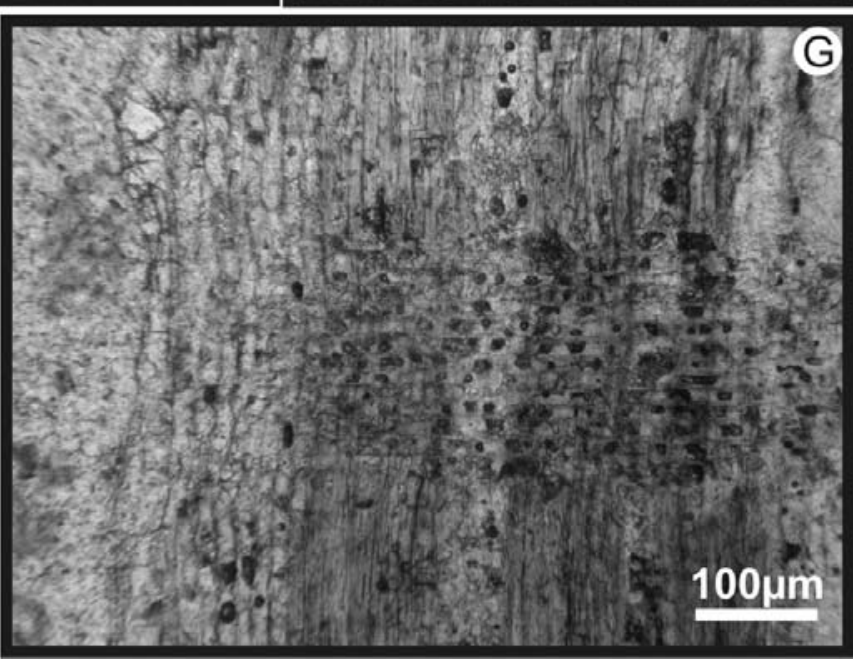

Figura 4 - A) Macrofotografia do exemplar NR570, lenho com tons claros de cinza. B) Imagem em Microscopia Eletrônica de Varredura, detalhes das pontoações intervasculares alternas e guarnecidas. C) Microfotografia em microscópio óptico de luz refletida, corte transversal, poro solitário de contorno arredondado e parênquima axial em faixas. Microfotografias em microscópio óptico de luz transmitida: D) Corte transversal destacando o parênquima em faixas longas de 3-7 células de largura; porosidade difusa; regiões claras indicam áreas não coradas pela safranina. E) Corte longitudinal radial, elementos de vasos curtos e placas de perfuração simples. F) Corte longitudinal tangencial com raios irregularmente estratificados, observa-se variação da fixação do corante. $G$ ) Corte longitudinal radial, corpo do raio com células procumbentes e uma fileira marginal de células quadradas. 

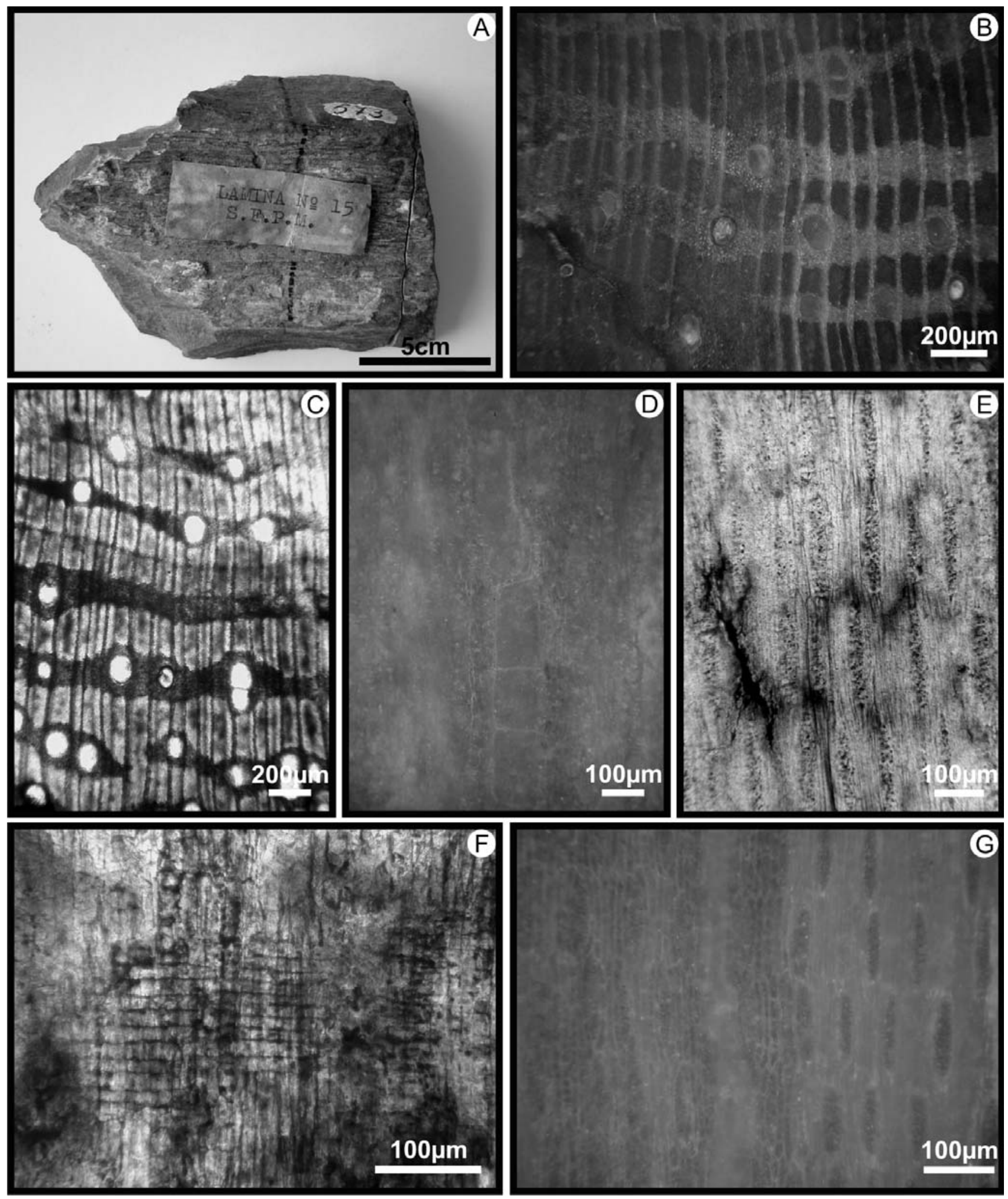

Figura 5 - A) Macrofotografia do exemplar NR573, lenho de cores escuras tendendo ao marrom. Microfotografias em microscópio óptico de transmissão ( $C, E$ e F) e de reflexão $(B, D$ e $G)$ do exemplar NR573. B e C) Cortes longitudinais transversal com parênquima axial paratraqueal aliforme confluente formando faixas, porosidade difusa, parênquima marginal. D) Corte longitudinal tangencial com elementos de vasos curtos e placas de perfuração simples. E e G) Corte longitudinal tangencial, estratificação dos raios, fibras e parênquima axial. F) Corte longitudinal radial, corpo do raio com células procumbentes e quadradas marginais. 
A comparação do exemplar fóssil com os exemplares da coleção de referência do Laboratório de Paleoecologia Vegetal do Museu Nacional e com dados da literatura apontou afinidades do mesmo com o gênero Zollernia (Fabaceae Caesalpinioideae).

\section{Família LYTHRACEAE}

[Figura 6]

MATERIAL EXAMINADO Brasil - Acre: Alto Purus, quase fronteira com o Peru. Gessé da Silva Maia 2001 (UFRJ-DG 746-Pb). Brasil - Acre: Rio Purus. Coleção Solms-Laubach (NR 491, DNPM-RJ).

DESCRIÇÃO MACROSCÓPICA Lenho de cor marrom-claro, cinza-claro a escuro (UFRJ-DG 746-Pb) ou de cor amarela a marrom-claro e escuro (NR 491). Em secção transversal, bandas claras de parênquima e poros visíveis a olho nu, sem arranjo preferencial, poros solitários na maioria e múltiplos de 2-3 radiais e aparentando tiloses. Anéis de crescimento indistintos a olho nu (NR 491) ou individualizados por faixas de parênquima marginal (746-Pb). Raios muito finos visíveis em lupa. Em secção longitudinal tangencial, raios baixos e numerosos, de difícil observação em lupa. Linhas vasculares longas, lineares com pequenas regiões irregulares, de cores claras e visíveis a olho nu.

DESCRIÇÃO MICROSCÓPICA Limite da camada de crescimento distinta, demarcado por parênquima marginal. Lenho com porosidade difusa. Vasos predominantemente solitários $(87,5 \%)$, geminados e múltiplos de dois $(10,5 \%)$ e raros múltiplos $(2 \%)$ dispostos em cachos. Todos os vasos solitários de contorno arredondado. Diâmetro tangencial do lúmen dos vasos em média de 330,1 $\mu \mathrm{m}$ (maior padrão de desvio $=71,5$; intervalo 196,4-494,1 $\mu \mathrm{m} ; \mathrm{n}=25)$. Muito poucos poros por milímetro quadrado (média $=1,8$; maior padrão de desvio $=1,0$; intervalo $0-4 ; \mathrm{n}=25$ ). Média do comprimento dos elementos de vasos de 496,3 $\mu \mathrm{m}$ (maior padrão de desvio $=89,0$; intervalo $314,6-658,5 \mu \mathrm{m} ; \mathrm{n}$ =7-25). Tilos frequentes. Placas de perfuração simples. Pontoações intervasculares alternas, guarnecidas, com diâmetro médio a grande (média $=9,6 \mu \mathrm{m}$; padrão de desvio $=1,8$; intervalo $6,8-14,2 \mu \mathrm{m} ; \mathrm{n}=25)(746-\mathrm{Pb})$ ou grande (média $=15,6 \mu \mathrm{m}$; padrão de desvio $=3,3$; intervalo 12,4-23,1 $\mu \mathrm{m} ; \mathrm{n}=8)(\mathrm{NR} 491)$. Parênquima axial paratraqueal vasicêntrico e aliforme, podendo ocorrer bandas marginais (NR 491). Largura do raio de 1-3 células, maioria bisseriados $(92,9 \%)$, poucos raios unisseriados $(6,2 \%)$ e, raros trisseriados $(0,9 \%)$. Raios de comprimento baixo (média $=446 \mu \mathrm{m}$; padrão de desvio $=111,2$ intervalo $215,1-790,1 \mu \mathrm{m} ; \mathrm{n}=24$ 25). Raios heterogêneos, multisseriados com corpo do raio com células procumbentes e uma ou duas fileiras marginais de células quadradas ou eretas, raios unisseriados compostos por células quadradas e eretas. Raios por milímetro em média 9,2 (padrão de desvio $=2,0$; intervalo 5-13; $\mathrm{n}=25-10$ ).

COMPARAÇÃO As características anatômicas mais relevantes taxonomicamente das amostras analisadas são parênquima aliforme associado a raios heterogêneos e presença de pontoações guarnecidas.

Apenas as famílias Fabaceae e Lythraceae reúnem o conjunto dos características distintivos encontrados. No entanto, raios heterogêneos são raros em Fabaceae e somente ocorrem em certos gêneros com características anatômicas especiais, por exemplo, Copaifera e Eperua, ambas apresentando longas linhas de canais axiais muito conspícuos (Détienne \& Jacquet 1983). Deste modo, os exemplares analisados podem ser atribuídos à família Lythraceae.

\section{Família MYRTACEAE}

[Figura 7]

MATERIAL EXAMINADO Brasil - Acre: Niterói $\left(10^{\circ} 08^{\prime} 14\right.$ ' $\left.\mathrm{S}, 67^{\circ} 48^{\prime} 46^{\prime \prime} \mathrm{W}\right)$, na margem esquerda do Rio Acre (Pb 168, LPP/UFAC).

DESCRIÇÃO MACROSCÓPICA Lenho de cor marrom-escuro. Fraturas preenchidas no sentido dos raios. Em secção transversal, poros visíveis a olho nu, sem arranjo preferencial, praticamente todos solitários sendo raros os múltiplos de 2. Parênquima não individualizado a olho nu ou em lupa. Células torcidas presentes. Secção longitudinal tangencial, raios não visíveis em lupa. Linhas vasculares lineares, longas e sem conteúdo.

DESCRIÇÃO MICROSCÓPICA Limite da camada de crescimento distinta, demarcada pela redução na frequência e diâmetro dos poros. Lenho com porosidade difusa. Vasos solitários $(90 \%)$ e geminados $(10 \%)$. Todos os vasos solitários de contorno arredondado. Diâmetro tangencial do lúmen em média de $122,7 \mu \mathrm{m}$ (padrão de desvio $=22,3$; intervalo 64,8-167,1 $\mu \mathrm{m} ; \mathrm{n}=25$ ). Média de 7,6 vasos por milímetro quadrado (padrão de desvio $=1,71$; intervalo $4-11 ; n=25$ ). Placas de perfuração simples, retas ou escalariformes, com mais de 10 barras, inclinadas. Pontoações intervasculares alternas, guarnecidas e de diâmetro médio (média $=4,7 \mu \mathrm{m}$; padrão de desvio $=1,2$; intervalo 2,4-7,6; $\mathrm{n}=25$ ). Parênquima axial apotraqueal difuso e em agregados. Raios muito frequentes, 2-3 seriados, com longos segmentos unisseriados nas extremidades; raios heterogêneos com células procumbentes e muitas fileiras marginais de células quadradas e eretas; raios com mais de $2 \mathrm{~mm}$ de altura. 

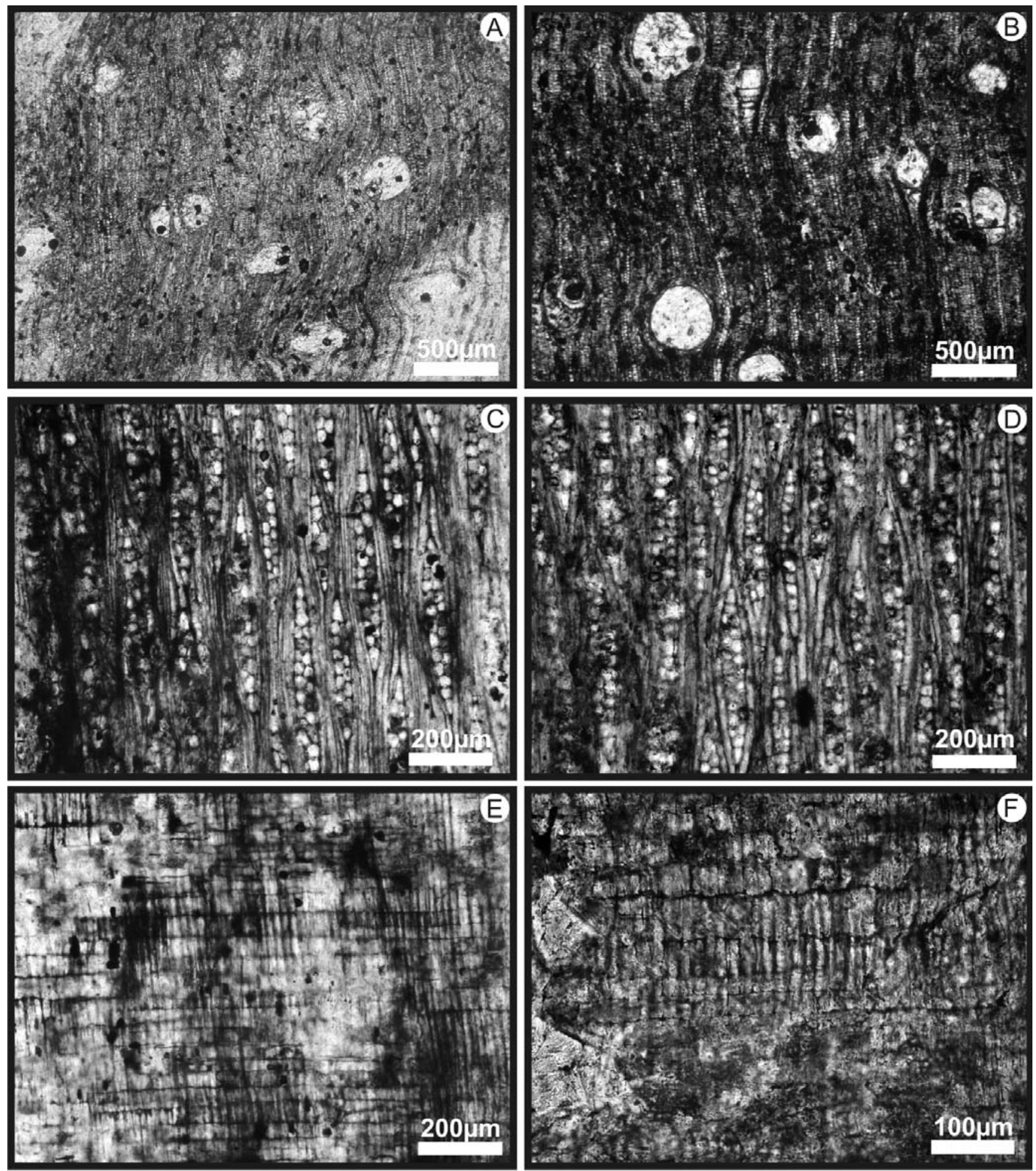

Figura 6 - A) Corte transversal do exemplar NR 491, DNPM-RJ. B) Corte transversal do exemplar UFRJ-DG 746-Pb. A-B. Porosidade difusa; parênquima axial paratraqueal aliforme; tiloses. C) Corte longitudinal tangencial do exemplar NR491. D) Corte longitudinal tangencial do exemplar Pb746. C-D. Raios numerosos (9 por milimetro linear) e baixos; poucos unisseriados, predominantemente bisseriados e raros trisseriados. E) Corte longitudinal radial do exemplar NR 491, DNPM-RJ. F) Corte longitudinal radial do exemplar UFRJ-DG 746Pb. E-F) Corpo dos raios constituídos por células procumbentes no centro e eretas ou quadradas nas margens. 

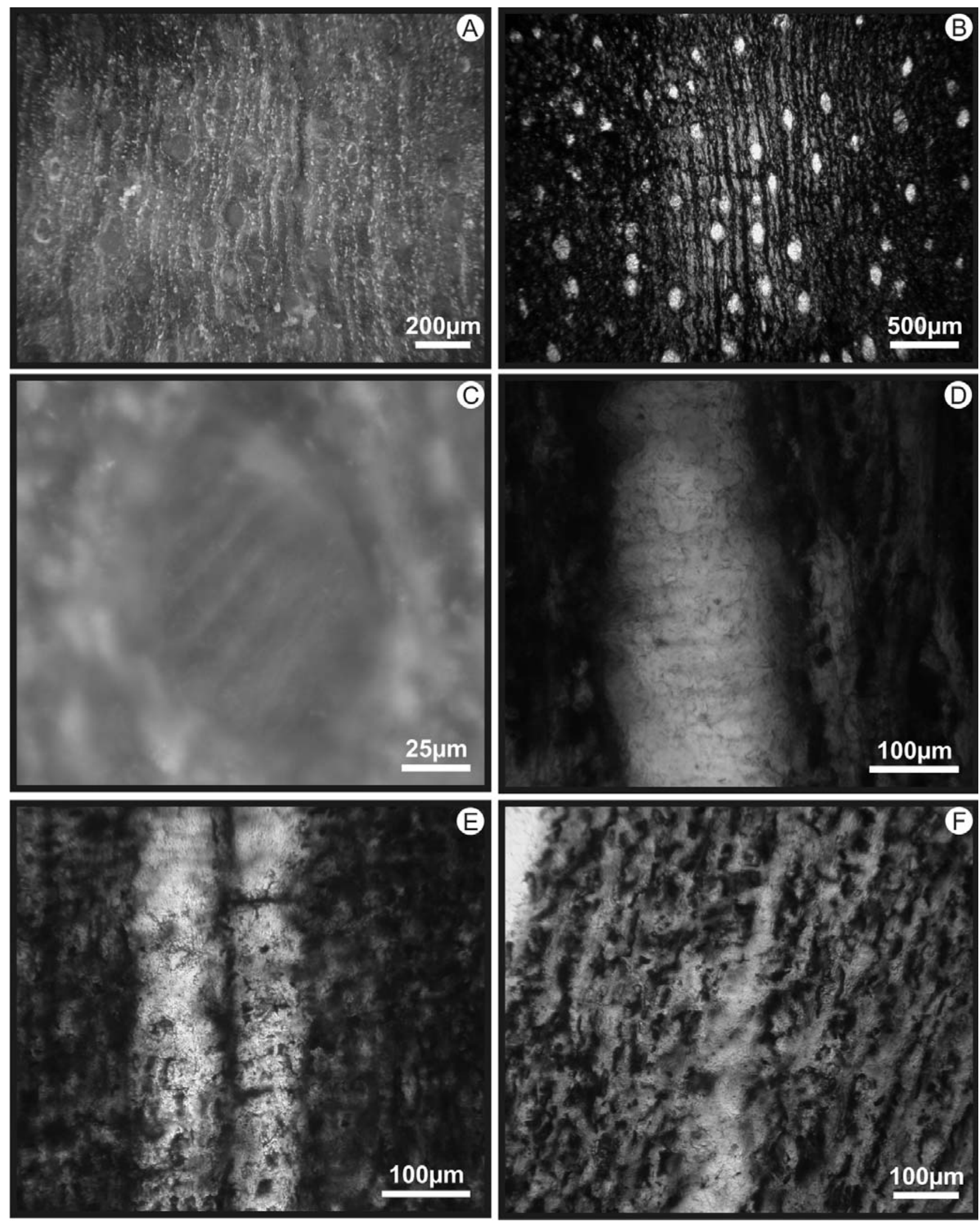

Figura 7 - Microfotografias em microscópio óptico de transmissão (B, $D, E$ e F) e de reflexão (A e C) do exemplar Pb168. A) Corte transversal, destacam-se as diferentes colorações da amostras, com regiões avermelhadas (safranina), amareladas (óxidos de ferro), e áreas claras (sílica). B) Corte transversal, observa-se a porosidade difusa, com poros predominantemente solitários, escala $500 \mu \mathrm{m}$. C) Placa de perfuração escalariforme em corte transversal. D e E) Placas escalariformes em corte longitudinal tangencial, inclinadas. F) Placas de perfuração simples, horizontais. 
COMPARAÇÃO As características anatômicas mais relevantes taxonomicamente da amostra analisada são: poros exclusivamente solitários, com arranjo tendendo a oblíquo, raios heterogêneos, parênquima difuso e em agregados, pontoações guarnecidas e placa de perfuração escalariforme.

As características anatômicas permitem atribuir o exemplar estudado a família Myrtaceae. A identificação de gêneros dentro da família Myrtaceae não pôde ser realizada pela grande dificuldade taxonômica em se distinguir os gêneros desta família, de um lado devido à grande homogeneidade da anatomia da madeira de suas espécies, e de outro, pela variabilidade interespecífica de certos gêneros, especialmente Eugenia (Détienne \& Jacquet 1983, Scheel-Ybert 1998). As características anatômicas observadas não permitem vincular a um gênero.

Comparação ecológica através da afinidade botânica atual Algumas características anatômicas apresentam relevantes aplicações para interpretações ambientais. Foram analisados os anéis de crescimento, agrupamento e arranjo dos poros, placas de perfuração e espessamento espiralado.

Os exemplares apresentam anéis de crescimento ou cernes demarcados por parênquima marginal e variação na frequência e diâmetro dos poros. A atividade cambial pode ser afetada pelo suprimento de água, temperatura, intensidade luminosa, fotoperíodo e gradiente hormonal (Palermo et al. 2002). Anéis de crescimento em espécies vegetais brasileiras foram observados em $48 \%$ dos lenhos estudados por Alves \& Angyalossy-Alfonso (2000). Segundo estes autores, a presença de anéis de crescimento tem uma relação negativa com ambientes superúmidos, confirmando a relação entre a contínua atividade cambial e climas tropicais úmidos. A ausência dos anéis de crescimento mostra uma relação estatística positiva com clima mediterrâneo. Contudo, a seca e a inundação em zonas tropicais, também induzem a inatividade fisiológica (Palermo et al. 2002). Vetter \& Botosso (1989) observaram o desenvolvimento dos anéis de crescimento em 30 espécies amazônicas atuais decorrentes de uma significante variação no suprimento de água.

São observados nos lenhos fósseis porosidade difusa, podendo ocorrer anéis semiporosos ou uma tendência a esta porosidade. Os poros são solitários e em múltiplos de dois, raramente de três. Alves \& Angyalossy-Alfonso (2000), constataram que $84 \%$ das espécies brasileiras analisadas apresentaram porosidade difusa. Anéis semiporosos estariam relacionados a altas latitudes e a ambientes sazonais (Alves \& Angyalossy-Alfonso 2000). Vasos múltiplos mostram uma relação positiva com a região sudeste e com a floresta sazonal semidecídua, e uma relação negativa com a região norte do Brasil (Alves \& Angyalossy-Alfonso 2000). Arnold \& Mauseth (1999), constataram que, em Cactaceae, o aumento da disponibilidade de água poderia induzir as plantas a produzir vasos maiores.

Os lenhos fósseis apresentam placas de perfuração simples, sendo em uma espécie escalariformes com menos de 10 barras. Alves \& Angyalossy-Alfonso (2000), encontraram placas de perfuração simples em $95 \%$ dos exemplares atuais estudados. Os autores reportaram que placas de perfuração múltiplas são raramente encontradas em florestas tropicais de baixas altitudes e são mais comuns em zonas frias, regiões temperadas árticas e em floras tropicais de alta montanha. Placas de perfuração múltiplas são mais comuns em climas temperados e mesotérmicos, caracterizados pela baixa temperatura durante o inverno. Segundo Wheeler (1991), placas de perfuração múltiplas são adaptações para ambientes com geada, dando a capacidade de evitar as bolhas do degelo. Contudo o embolismo pode ser resultado da seca fisiológica, em ambientes com temperaturas que não são baixas suficientes para o congelamento.

Os exemplares fósseis estudados não apresentam nenhum tipo de espessamento. Os espessamentos espiralados são geralmente encontrados em floras temperadas e são raros em floras tropicais (Carlquist 1975).

CONCLUSÕES As características anatômicas dos lenhos fósseis indicam afinidades botânicas com as famílias Fabaceae Caesalpinioideae, Lythraceae e Myrtaceae.

A análise da preservação dos elementos constituintes do lenho possibilitou reunir dados de natureza tafonômica. Os exemplares encontram-se permineralizados, sendo a sílica o principal mineral constituinte dos lenhos, conforme observado também por Mussa (1959). Também são observadas inclusões de óxidos de ferro e matéria orgânica residual. Há diferentes graus de preservação celular, resultantes dos processos tafonômicos ocorridos antes e após o sepultamento.

Considerações paleoclimáticas e paleoambientais foram estabelecidas através da anatomia ecológica da madeira. O conjunto das características anatômicas observadas em cada exemplar possibilitou inferir um clima quente e úmido para a região do Acre durante o Mioceno final. A presença de anéis de crescimento em todos os espécimes sugere uma variação na disponibilidade hídrica, indicando clima sazonal.

Assim, o estudo dos lenhos fósseis da Formação Solimões demonstrou a importância como indicadores paleombientais para a região Norte do Brasil.

Agradecimentos Ao Conselho Nacional de Desenvolvimento Científico e Tecnológico (CNPq) e FAPERJ pelo apoio financeiro ao desenvolvimento do projeto. 
Ao Programa de Pós-graduação em Geologia, Departamento de Geologia, Instituto de Geociências, Centro de Ciências Matemáticas e da Natureza, da Universidade Federal do Rio de Janeiro. A Dra. Rita de Cassia Tardin
Cassab, do Departamento Nacional de Produção Mineral, Ministério das Minas e Energia, Museu de Ciências da Terra $9^{\circ}$ Distrito RJ, pelo empréstimo dos exemplares para o estudo.

\section{Referências}

Alvarenga H.M.F. \& Guilherme E. 2003. The Anhingas (Aves: Anhingidae) from the upper Tertiary (MiocenePliocene) of Southwestern Amazonia. Journal of Vertebrate Paleontology, 23(3):614-621.

Alves E.S. \& Angyalossy-Alfonso V. 2000. Ecological trends in the wood anatomy of some Brazilian species. I: Growth rings and vessels. IAWA Journal, 21(1):3-30.

Arnold D.H. \& Mauseth J.D. 1999. Effects of Environmental Factors on Development of Wood. American Journal of Botany, 86(3):367-371.

Baretta-Kuipers T. 1981. Wood anatomy of Leguminosae: its relevance to taxonomy. In: Polhill R.M. \& Raven P.H. (ed.). Advances in Legume Systematics. Royal Botanic Gardens, Kew Publishing, p. 677-705.

Bezzera P.E.L. 2003. Compartimentação morfotectônica do interflúvio Solimões-Negro. Tese de Doutorado, Universidade Federal do Pará, Belém, 335 p.

Bocquentin J. \& Guilherme E. 1997. A cintura pélvica do quelônio Stupendemys (Podocnemididae, Podocnemidinae) proveniente do Mioceno Superiorplioceno, do Estado do Acre, Brasil. Estudos tecnológicos, Acta Geol. Leopoldensia, 20(45):47-50.

Bolzon R.T. 1999. Lenhos de Dicotyledoneae do Cenozóico da Região de Uruguaiana, Estado do Rio Grande do Sul, Brasil. Tese de Doutorado, Universidade Federal do Rio Grande do Sul, Porto Alegre, 199 p.

Bolzon R.T. \& Marchiori J.N.C. 2002. Lenhos fósseis de Myrtaceae da região de Uruguaiana, Estado do Rio Grande do Sul, Brasil. Acta Geologica Leopoldensia, 25(54):41-63.

Carlquist S. 1975. Ecological strategies of xylem evolution. Berkely, University of Califórnia Press, 259 p.

Cavalcante L.M. 2005. Zoneamento geológico e geomorfológico entre Feijó e Mãncio Lima - Acre. Rio Branco, Embrapa, Documentos 99, 24 p.

Cavalcante L.M. 2006. Aspectos geológicos do estado do Acre e implicações na evolução da paisagem. Rio Branco, Embrapa, Documentos 104, 25 p.

Cozzuol M.A., Goin F., Reyes M., Ranzi A. 2006. The oldest species of Didelphis (Mammalia, Marsupialia, Didelphidae), from the late Miocene of Amazonia. Journal of Mammalogy, 87(4):663-667.

Cruz N.M.C. 1984. Palinologia do Linhito do Solimões no Estado do Amazonas. In: MME/DNPM, Symposium Amazonico, 2, Anais, p. 473-480.

Daemon R.F. \& Contreiras C.J.A. 1971. Zoneamento palinológico da Bacia do Amazonas. In: SBG, Congresso Brasileiro de Geologia, 25, Anais, p. 79-91.

Del Corro G. 1964. La Gondwania el Antiguo Continente Austral. Revista Del Museu Argentino de Ciências
Naturales "Bernardino Rivadavia", 12:1-90.

Détienne P. \& Jacquet P. 1983. Atlas d'identification des bois de l'amazonie et dês regions voiseines. Paris, Centre Technique Forestier Tropical, $640 \mathrm{p}$.

DNIT - Departamento Nacional de Infraestrutura de Transportes. 2002. Mapa rodoviário do Estado do Acre. Disponível em: http http://www2.transportes.gov.br/ bit/01-inicial/01-estadual/estados/port/ac.htm. Acessado em: 23/01/2012.

Feijó F.J. \& Souza R.G. 1994. Bacia do Acre. Boletim de Geociências da Petrobrás, 8(1):9-16.

Frailey C.D., Lavina E.L., Rancy A., Souza Filho J.P. 1988. A proposed Pleistocene/Holocene lake in the Amazon Basin and its significance to Amazonian Geology and Biogeography. Acta Amazonica, 18(3-4):119-143.

Hass H. \& Rowe N.P. 1999. Thin sections and wafering. In: Jones T.P. \& Rowe N.P. (eds.) Fossil Plants and Spores. Londres, Geological Society, p. 76-81.

Hoorn C. 1993. Miocene incursions and the influence of Andean tectonics on the Miocene depositional history of northwestern Amazonian: results of a palynostratigraphic study. Palaeogeography, Palaeoclimatology, Palaeoecology, 105:267-309.

Kay R.F. \& Cozzuol M.A. 2006. New platyrrhine monkeys from the Solimões Formation (late Miocene, Acre State, Brazil). Journal of Human Evolution, 50:673-686.

Latrubesse E.M., Bocquentin J.C., Santos J.C., Ramonell C. 1997. Paleoenvironmental Model for the Late Cenozoic of Southwestern Amazonia: Paleontology and Geology. Acta Amazonica, 27(2):103-118.

Latrubesse E.M., Silva S.A.F., Cozzuol M., Absy M.L. 2007. Late Miocene continental sedimentation in southwestern Amazonia and its regional significance: Biotic and geological evidence. Journal of South American Earth Sciences, 23:61-80.

Maury C.J. 1937. Argilas fossilíferas do Plioceno do Território do Acre. Boletim do Serviço Geológico e Mineralógico do Brasil, 77:1-29.

Miura K. 1972. Possibilidades petrolíferas da Bacia do Acre. In: SBG, Congresso Brasileiro de Geologia, 26, Anais de Resumo, p. 15-20.

Mussa D. 1959. Contribuição à paleontologia vegetal: II madeiras fósseis do território do Acre (Alto Juruá) Brasil. Boletim do Departamento Nacional de Produção Mineral, 195:1-54.

Mussa D., Bernades-De-Oliveira M.E., Mune S.E., De Siqueira E., Castro-Fernandes M.C. de, Carmo D.A. do 2002. Lenho de Dicotiledônea da Formação Solimões, Neógeno da Bacia do Acre, Brasil. Paleontologia em Destaque, 40:16-17. 
Norton O.R. \& Curchin J. 2002. Basic of Polarized Light Microscopy: Part 1. Meteorite, 8:26-27.

Oliveira M.R.S. \& Souza Filho J.P. 2001. Distribuição geográfica da família Nettosuchidae (Crocodyliformes) na Amazônia Sul-Ocidental (Estado do Acre). In: SBP, Congresso Brasileiro de Paleontologia, 17, Boletim de Resumos, p. 150.

Palermo G.P.M., Latorraca J.V.F., Abreu H.S. 2002. Métodos e Técnicas de Diagnose e de Identificação dos Anéis de Crescimento de Árvores Tropicais. Floresta e Ambiente, 9(1):165-175.

Passos V.T.R. 2000. Geomorfologia. Rio Branco, SECTMA, Relatório Técnico, p. 22-29.

Petri S. \& Fúlfaro V.J. 1983. Geologia do Brasil (Fanerozóico). São Paulo, Editora T.A. Queiroz/EDUSP, 631 p.

Ramos M.I.F., Silveira, R.R., Coimbra J.C. 2001. Ostracodes mixoalinos do Mioceno da Formação Solimões, Bacia do Solimões, Amazonas, Brasil In: SBP, Congresso Brasileiro de Paleontologia, 17, Rio Branco, Boletim de Resumos, p. 86.

Räsänen M., Linna A., Irion G., Hernani L.R., Huanan R.V. Wesseling F. 1998. Geologia e geoformas da área de Iquitos. In: Kalliol S.F. \& Paitán S.F. (eds.) Geologia e dessarollo em la zona de Iquitos, Peru. Turku, Turun Yliopisto, p. 21-95.

Richter M.1984. Dental histology of a Characoid fish from the Plio-Pleistocene of Acre, Brazil. Zoologica Scripta, 13(1):69-79.

Richter M. 1989. Acregoliathidae (Osteichthyes, Teleostei), a new family of fishes from the Cenozoic of Acre State, Brasil. Zoologia Scripta, 2(18):311-319.

Scotese C.R. 2003. Paleomap Project. Disponível em: http:// www.scotese.com. Acessado em: 30/09/2010.

Scheel-Ybert R. 1998. Stabilité de l'Écosystème sur le Littoral Sud-Est du Brésil à l'Holocène Supérieur (5500-1400 ans BP). Les Pêcheurs-Cueilleurs-Chasseurs et le Milieu Végétal: Apports de l'Anthracologie. Thèse de Doctorat, Université Montpellier II, Montpellier, 520 p.

Silva S.A.F., Absy M.L., Dino R., Antonioli L. 2004. Dados palinoestratigráficos da Formação Solimões em amostras coletadas em sítios fossilíferos no Estado do Acre. In: ALPP, Reunião de Paleobotânicos e Palinólogos, 11, Boletim de Resumos, p. 140.

Souza Filho J.P. 1998. Novas formas fósseis de Crocodylia (Alligatoridae e Gavialidae) da Formação Solimões, Cenozóico do Estado do Acre-Brasil, representadas por materiais cranianos e mandibulares. Dissertação de Mestrado, Instituto de Geociências, Universidade Federal do Rio Grande do Sul, Porto Alegre, 194 p.

Vetter R.E. \& Botosso P.C. 1989. Remaks on age and growth rate determination of Amazonian trees. IAWA Bulletin: New Series, 10(2):133-145.

Vonhof H.B., Wesselingh F.P., Ganssen G.M. 1998. Reconstruction of the Miocene western Amazonian aquatic system using molluscan isotopic signatures. Palaeogeography, Palaeoclimatology, Palaeoecology, 141:85-93.

Wheeler E.A. 1991. Fossil dicotyledonous wood database, CA LaPasha (programed), Computer Assisted Wood Identification, version 1.1. Raleigh, North Carolina State University, 1991.

Wheeler E.A. \& Baas P. 1992. Fossil wood of the Fabaceae; A case study in xylem evolution and ecological anatomy. In: Herendeen P.S. \& Dilcher D.I. (eds.) Advances in Legume Systematics Part 4. The Fossil Record. Royal Botanic Gardens, Kew Publishing, p. 281-301.

Manuscrito ID 19933

Submetido em 20 de novembro de 2010 Aceito em 07 de fevereiro de 2012 